\title{
Effects of Educational Interventions to Improve Safe Hazardous Drug Handling among Oncology Nurses: Systematic Review
}

\author{
Salam Banihani ${ }^{1}$, SakhaaHabashneh $^{2}$, Aida Suhemat ${ }^{3}$, EmanAwabdeh $^{3}$, Jafar A. Alshraideh $^{4}$ \\ ${ }^{1}$ Clinical Instructor, Department of Adult Health Nursing, Faculty of Nursing, Al al-Bayt University, Mafraq, \\ Jordan, ${ }^{2}$ Lecturer, Department of Adult Health Nursing, Faculty of Nursing, Mutah University, Karak, Jordan, \\ ${ }^{3}$ PhD Student, ${ }^{4}$ Professor, School of Nursing, The University of Jordan, Amman, Jordan
}

\begin{abstract}
Background: Oncology nurses are in the front line of handling Hazardous Drugs (HDs) in health care settings.It is crucial to minimize the cytotoxic effects of handling HDs among oncology nurses by following the safety standards practices, protocols, and guidelines. The purpose of this systematic review is to identify the effects of educational interventions to reduce exposure of hazardous drugs and chemicals among oncology nurses.

Methods: The review was performed following the Preferred Reporting Items for Systematic Review and Meta-Analysis (PRISMA) guidelines. The search protocol was conducted using PubMed MEDLINE, CINAHL, and Google Scholar, for studies published between the periods of 2013to 2021. Nine articles were included in the systematic review

Conclusion: Severalinterventions were identified including web-based online courses, educational modules, applied safety protocols, demonstration and re-demonstration, videos, PowerPoint presentations, seminars, and workshops. Following up nurses after applying different educational interventions showed enhanced adherence to safe handling of HDs. Applied educational interventions have been reported to improve self-efficacy, nursing performance, attitude, knowledge, and practice of cytotoxic drug handling. A better compliance was reported on nurses'use of standard personal protective equipment, safety protocol during administration, handling of body fluids, and responding to spills of HDs.
\end{abstract}

Keywords: Hazardous drugs; Occupational health work; Oncology nurses; Safe handling

\section{Introduction}

Contamination of the work environment with Hazardous Drugs (HDs), increases the potential

\section{Corresponding author:}

\section{Salam Banihani,}

RN, MSN (orcid.org/0000-0003-0848-5615)

Clinical Instructor Department of adult health nursing, Faculty of Nursing Al al-Bayt University, Mafraq, Jordan, E-mail: banihani.salam@yahoo.com exposure of health care workers including nurses if they were not handled appropriately ${ }^{[1]}$. Hazardous Drugs include chemicals used for cancer therapy that is known as a carcinogen, teratogen, and reproductive toxin. It also includes some antiviral drugs, hormonal agents, and other new drugs that mimic HDs in structure or the level of toxicity ${ }^{[2]}$. Work-related exposure to HDs has been associated with acute symptoms on different body systems including but not limited to hair loss, contact dermatitis, allergic reaction, abdominal cramps, eye injury, reproductive 
problems such as infertility, miscarriage, abortion, and fetal anomalies ${ }^{[3]}$.

Exposure to HDs can occur during dispensing, compounding, and administering the drugs. Aerosols as well maybe spreading during HDs preparation and administration. ${ }^{[4]}{ }^{[5]}$. Careful handling of such HDs is crucial to minimize the adverse events of exposure to toxic substances and reduce contamination in health care settings ${ }^{[1]}$.

Compliance with the safety precautions including Personal Protective Equipment (PPE), training and education about the handling of HDs, using of engineering control to isolate the toxic effect in health care settings are examples of precautions that could reduce occupational exposure to HDs [6]'[7]. Studies on adherence of nurses with safe handling of HDs has been reported ${ }^{[8,9]}$, however limited studies have specified factors that could actually reduce the serious side effects of exposure to HDs. Several factors were known to be the leading cause of inappropriate handling of HDs including lack of knowledge about risk ${ }^{[10]}$, false perceiving of risks, self-efficacy to use PPE ${ }^{[11]}$, and organizational factors ${ }^{[12]}$.

\section{purpose}

The purpose of this systematic review is to assess the effects of educational interventions to improve safe HDs handling among oncology nurses.

\section{Material and Methods}

\section{Search Criteria and Study Selection}

This review was conducted and reported according to the Preferred Reporting Items for Systematic Review and Meta-Analysis (PRISMA) guidelines ${ }^{[13] .}$ To answer the research question, PICOT framework was used. The components include Population of interest (P), Issue of interest (I), Comparison of interest (C), Outcome of interest (O), and Time frame $(\mathrm{T}){ }^{[14]}$. Our review focus on oncology nurses
(P), educational intervention to improve safe HDs handling (I), comparison of interest (C) was not relevant. In relation to outcome, it is hypothesized that educational intervention will improve safe HDs handling among oncology nurses in occupational health work $(\mathrm{O})$. The time frame was set to include published articles from the period from 2010 to 2021 (T). The guiding question for the current systematic review is: what are the effects of an educational interventions to improve safe handling of HDs and chemicals among oncology nurses?

The retrieved articles were included if they were: (1) primary research reports, (2) published between 2010 and 2021, (3) related to the effect of educational intervention on safe handling of HDs and chemicals among oncology nurses, (4) written in English, (5) quantitative studies including controlled trials, cross-sectional, quasi-experimental, case-control, and cohort designs. However, (1) Studies before 2010, (2) articles in languages other than English, (3) editorial, reviews, and experts' reports were excluded. Four nurse researchers conduct the searches in four different databases independently. Cumulative Index of Nursing and Allied Health Literature (CINAHL), Medline through (PubMed), Science Direct, and Google Scholar. The Initial search was started on March $1^{\text {st }}, 2021$ and the last search was run out on April 1 ${ }^{\text {st }}, 2021$.

\section{Study Coding}

Terms were entered according to medical subject heading $(\mathrm{MeSH})$ to identify research articles. Initially, broad categories were searched such as nurses, health care workers and chemotherapy handling. Then, more specific keywords were searched to include "Hazardous drugs", “education”, “occupational health" and "oncology nurse". Additionally, these words were searched in combination with each other to identify other publications. Reference lists of eligible studies were also searched. Two researchers 
screened the retrieved articles by titles and abstracts independently, to identify studies that will be included in the review. The full text of studies that were found to be eligible was retrieved and assessed to ensure eligibility. Disagreement over the eligibility of studies was resolved through discussions and by a third reviewer when necessary. Data were extracted by two reviewers using a special extraction sheet developed by researchers. Data were included if consensus was reached between the reviewers.

\section{Analysis}

Data were extracted and summarized in two tables: (1) a summary of studies characteristics (including research methodology, interventions, settings, population, results, and recommendations), and (2) studies' findings that were summarized in a review matrix, with the following headings: author, year and country, title, objectives, design, settings and sample characteristics, findings, recommendations, and additional comments, respectively. Retrieved systematic reviews were assessed using the Critical Appraisals Skills Program (CASP) Checklist [15]. Quantitative studies were assessed using the Quality Assessment Tool (QAT) checklist ${ }^{[16]}$. Two reviewers assessed the risk of bias in the selected studies independently by using the Effective Public Health Practice Project (EPHPP) [17]. In case of different scores, agreement by all reviewers was achieved. Data were analyzed and synthesized by two reviewers, any disagreement between them was resolved by consensus. data were included only if both reviewers agreed.

\section{Results}

A total of (80) articles were retrieved. Fifty-seven remain after removal of duplicates and were screened by titles and abstracts. There were (18) relevant articles that underwent full-text screening of which nine were found eligible to be included in the review.

\section{Risk of Bias within Studies}

Trustworthiness checklists were used to assess and appraise the included studies. QAT was used to evaluate the quality of quantitative studies. This was interpreted through referring to the tool dictionary and a research study that examined quality assessment components and rating for EPHPP instrument [18]. Two studies indicate strong and fair on controlling for selection bias. Two studies used randomized clinical trials design, three used quasi-experimental design, and three studies used pre-test post-test. Only one study addresses the confounding variables. One study was blinded. All studies mention the reliability and validity of the data collection tool except for one study. Three studies described the drop-out and withdrawal of participants. Details of appraisal is presented in table 1

Table 1: Effective Public Health Practice Project (EPHPP) Quality Assessment Tool for quantitative

\begin{tabular}{|c|c|c|c|c|c|c|c|c|c|}
\hline \multicolumn{10}{|c|}{ studies $(n=9)$} \\
\hline & $\underline{\Xi}$ & ᄅ્ & $\overline{\bar{\Xi}}$ & $\bar{\Xi}$ & $\overline{\underline{\Xi}}$ & $\underset{\Xi}{\Xi}$ & $\stackrel{\bar{g}}{\underline{\Xi}}$ & $\stackrel{\bar{D}}{\underline{1}}$ & $\underset{\Xi}{\mathbb{I}}$ \\
\hline 1. Selection Bias & NA & NA & NA & 1 & NA & 2 & NA & NA & NA \\
\hline \multirow{2}{*}{$\begin{array}{l}\text { Study 2. Study design } \\
\text { 3. Control for confounders }\end{array}$} & 1 & 2 & 2 & 1 & 2 & 2 & 2 & 2 & 2 \\
\hline & NA & NA & NA & NA & NA & 2 & NA & NA & NA \\
\hline 4. Blinding & NA & NA & NA & 2 & NA & NA & NA & NA & NA \\
\hline 5. Data Collection Methods & 2 & 1 & 1 & 1 & 1 & 1 & 1 & 1 & 1 \\
\hline
\end{tabular}


Cont... Table 1: Effective Public Health Practice Project (EPHPP) Quality Assessment Tool for quantitative studies $(n=$

\begin{tabular}{|c|c|c|c|c|c|c|c|c|c|}
\hline \multirow{3}{*}{$\begin{array}{l}\text { 6. Withdrawals and Drop- } \\
\text { Outs. } \\
\text { 7. intervention integrity } \\
\text { 8.analysis }\end{array}$} & 1 & NA & NA & 1 & 1 & NA & NA & NA & NA \\
\hline & 1 & 1 & 1 & 1 & 1 & 1 & 1 & 1 & 1 \\
\hline & 1 & 1 & 1 & 1 & 1 & 1 & 2 & 1 & 1 \\
\hline
\end{tabular}

\section{Results of Individual Studies}

Nine studies were extracted and included in the current study, they were published between 2013 and 2021. Of those articles, $(n=4)$ had quasi-experimental design, ( $\mathrm{n}=3)$ used one group pre-post-test design, and $(n=2)$ were randomized control trails. A number of educational interventions were used to improve the safe handling of HDs among oncology nurses. Four studies used multidimensional intervening aids including demonstration and re-demonstration of safe practices of HDs handling, group discussion, seminar, audiovisual aids, and PowerPoint presentations. Two studies used web-based intervention courses. Two other studies applied safety protocols, and one study used standard educational module.

\section{Identification of commonly used interventional} practices to improve safe HDs handling among oncology nurses In the extracted articles, multidimensional interventions were used to improve the safe handling of HDs and cytotoxic agents in occupational health-related work. Mohsen and Fareed ${ }^{[25]}$, conducted a quasi-experimentalstudy, applying a chemotherapeutic safety protocol for a two-month period among oncology nurses $(n=63)$. The participants were assigned to two groups, in two different locations, one group consisting of $(n=45)$ and the other of $(n=18)$ nurses. Both groups received a formal booklet protocol that included information regarding chemotherapy, time of appearance of chemotherapy hazards, and ways of protection during handling, administration, and /or dealing with patient's excretion. Moreover, the protocol included the way that nurses should deal with drug splashes on the eye or skin and ways of cleaning solid surfaces from chemotherapy contamination.

The second quasi-experimental study that was conducted by Shetaia, Shereif [21], utilized a safety protocol that was composed of four phases; intervention assessment, planning, implementation, and evaluation. The researchers allocated the 45 oncology nurse participants into nine small groups, each group consisting of five nurses, receiving five sessions each lasting from 30 to 45 minutes. Different methodologies were used in each session including chemotherapy preparation, routes of administration, side effects, patient's preparation before and during the administration of a dose, and precautions and safe handling measures during and after chemotherapy administration.

Keat, Sooaid [20], applied multidimensional pharmacist-based interventions for $(n=96)$ nurses in their prospective interventional study. The interventions included a series of technical, educational, and administrative support measures like the initiation of a closed-system cytotoxic drug reconstitution (CDR) services, courses, training workshops, and guideline updates. Pharmacy CDR service using closed system was initiated during the intervention period. Moreover, cytotoxic drugs were readily prepared in used-form with drug-specific 
labels containing clear handling instructions instead of sending it undiluted to the wards. Furthermore, two sessions of continuous nursing education and safe handling of cytotoxic drug workshop were conducted.

Another multidimensional educational intervention study was performed by Aebersold, Kraft ${ }^{[24]}$ used multiple educational approaches such as online modules, in-person interactive sessions, and simulation activities in their paired one-group preposttest study. Study participants included oncology registered nurses $(n=292)$ and $(n=82)$ pharmacists.

Two Randomized Controlled Trials (RCTs) applied educational web-based interventions. In the first study that was performed by Friese, Yang ${ }^{[22]}$, the recruited study participants were randomly assigned to the control group $(\mathrm{n}=136)$ and intervention group $(n=121)$. The control group received access to an hour of educational module on the project website which summarized principles of safe HDs handling. On the other hand, the intervention group received a tailored intervention of as many as three short videos that addressed the barriers to Personal Protective Equipment (PPE) and an hour of educational module as the control group. Then, the intervention group reported chemotherapy drug spills they experienced during the study period and submitted plasma samples for analysis.

The second RCT study was conducted by Mun and Hwang [19], where they established a content composed of five basic and advanced learning modules for anticancer chemotherapy which are comprehensive cancer diagnosis and treatment, general principles of chemotherapy, chemotherapy drug safety management, intravenous maintenance management, and chemotherapy drug side effect management. The total learning time for those modules was 80 minutes.
Shetaia, Shereif ${ }^{[21]}$ handled a study using one group pre-test and post-test study. Fifty participants were allocated to two groups $(n=24,26)$ respectively. The educational package was developed according to the identified needs from the pre-test interventions and available literature. After the establishment of base-line data in the pre-test phase, the educational intervention took place using LCD media players as teaching-learning resources. Data were collected after four weeks of the intervention as a post-test intervention.

SAMIR, GARAS ${ }^{[26]}$, conducted a time-series quasi-experimental study to evaluate the effect of a designed teaching program on safe handling of chemotherapy among $(n=30)$ oncology nurses. The study sample was divided into five subgroups, equally, and randomly. The teaching program was implemented on ten sessions that covered both knowledge and related skills of safe handling of HDs. The estimated time for each session was 30 minutes approximately; the total time required for the whole program was 25 hours distributed over 50 days (three days/week). Then, the designed teaching program was implemented through seminars, group discussion, and demonstration/re-demonstration using audiovisual aids such as booklets, videos, and PowerPoint presentations.

In another quasi-experimental study, Crickman and Finnell [23] utilized a toolkit of intervention consisting of hazard identification, standardization of PPE, and education. These strategies were identified as evidence-based control measures to reduce occupational exposure. Standardization of PPE involved selecting gloves, face masks with eye shields, and chemotherapy-tested gowns. Visual tools were used to alert staff about handling of bodily fluids, responding to spills and PPE that is required for chemotherapy administration. Education was offered using an online educational video shared with 
all nurses on the oncology unit. The program was created by the researcher himself and lasted around 30 minutes.

Evaluation of the effect of educational intervention on safe handling of HDs among oncology nurses.

Among the selected studies in the current review, the researchers examined the effects of educational interventions to improve safe handling of HDs and cytotoxic agents among oncology nurses. Four studies presented a positive effect and improvement in knowledge and practice regarding the safe handling of HDs [20, 21, 25, 26]. Three studies also disclosed increased knowledge about safe handling of HDs [21, 23, 24].

According to the result of the RCT study, it was found that web-based self-learning serves as an effective learning strategy to enhance nurses' safe handling of anti-cancer chemotherapy [19]. On the other hand, another RCT study found that web-based interventions did not improve personal protective equipment (PPE) adherence among oncology participants. This is related to suboptimal content in the intervention, too few interactions with participants compared to the actual educational intervention, and such structural barriers to adopting desired behaviors [22].

\section{Discussion}

\section{Summary of evidence}

Seemingly, this review offers a summary of the studies that examined the effects of the educational interventions to improve the safe handling of HDs among oncology nurses. Many studies were conducted in the field of knowledge, attitude, and perception of safe handling of HDs, but little evidence was introduced in relation to the effects of educational intervention on nurse's compliance with the safe handling of HDs and cytotoxic agents in occupational health-related work. $33.3 \%$ of included studies were conducted in the USA [22-24]. The same percentage was conducted in Egypt $[21,25,26]$. Only one study used a theoretical framework to guide the intervention; however, they haven't clearly defined how it guided the study, despite the importance of the framework role to develop interventional studies to establish a clear relationship among the variables [19]. Most studies $(88.9 \%)$ revealed a strong association between educational interventions and the safe handling of HDs. However, one study (11.1\%) showed that both online and web-based educational interventional courses did not improve participant's performance. This could be due to suboptimal interventional content, little interaction with participants compared to traditional teaching methods, and structural barriers to achieve desired behaviors ${ }^{[22,25]}$.

$44.4 \%$ of the extracted studies used multidimensional interventions such as group discussion, seminars, audiovisual aids, online modules, and simulations as a methodology for educational interventions. An example of an effective strategy may be identified through Keat, Sooaid [20] study for educational effectiveness to improve safe HDs handling among oncology nurses in Malaysia. It is a pharmacist-based prospective intervention to assess the variation of nurses' safety related to knowledge, attitude, and HDs handling practice in clinical wards after a series of multidimensional interventions including technical, administrative, and educational measures consisting of institution of closed-system cytotoxic drug reconstitution (CDR), courses, training workshops, and guideline updated. The findings reveal the effectiveness of education and training to improve knowledge on HDs handling, cytotoxic hazardous effect, way of exposure to HDs, use of PPE, and safe handling measures. Moreover, after applying the intervention the results showed improvement of nurses' attitude towards certain issues related to safety, and confidence levels when handling 
HDs. Four studies (44.4\%) were longitudinal studies that evaluated the effectiveness of HDs safe handling that were conducted after more than 3 months of educational interventions. This limits the ability to draw conclusions about the sustainability of the intervention's effectiveness ${ }^{[22,24,26,27]}$. Another point is that quasi-experimental studies lack randomization which introduces a selection bias that could affect the strength of intervention effectiveness among participants $[21,23,25,26]$. A single group pre-test and post-test design was applied in $66.7 \%$ of studies to assess the effect of educational intervention which may affect the internal validity because of not comparing with oncology nurses who were not exposed to the educational intervention [20, 21, 23, 24, 26, 27].

\section{Limitation}

Limitation of the review is the restriction of English language publications, low numbers of enrolled studies according to eligibility criteria, and heterogeneity of the included studies' design.

\section{Conclusion}

Multi-dimensional educational training programs produce strong evidence of positive effects on improving nurses' knowledge, performance, selfefficacy, attitude, and practice of cytotoxic drug handling. Outcomes include increased compliance with the standard of nurse's PPE and safety protocol during administration, safety handling measures, drug preparation, transportation, administration, discontinuation, waste disposal, spillage management, and care of safety cabinet. To minimize the risk of HDs exposure, health care workers must receive adequate training and equipment. Policymakers, clinical experts, and health system leaders should encourage clinical settings to adopt updated chemotherapy safety protocols concordant to PPE policies and activities. Further research on technical items and procedures that shape the educational program is needed to identify the structures that have value for clinical results.

Implications for Future Practice, Policy, and Research

Nurses are the front liners using HDs and other cytotoxic agents. They must have adequate knowledge and practice regarding the safe handling of HDs. This could be achieved by establishing a periodic training session including multi-dimensional educational interventions to improve knowledge, practice, and performance of safe handling of HDs. Using PPE to enhance safe workplace climate should be encouraged. Further studies are required to evaluate these interventions implications, contextual factors influencing the outcomes, and clear relationships between intervention components and outcomes.

Evidence-based development of policies should be tackling the barriers that inhibit the effectiveness of educational interventions. More attention should be given to the cost-effectiveness of interventions. Managing the environment is necessary to improve protection of oncology nurses when handling and administrating HDs. Moreover, recognizing inhibiting factors related to incompliance of oncology nurses to the guidance of HDs handling such as high workload, lack of time, unavailability of educational intervention, and infrequent updating of the protocol are recommended.

\section{References}

1. Randolph, S.A., Hazardous drugs: handling in health care settings. Workplace health \& safety, 2018. 66(5): p. 264-264.

2. Lombardo, J. and C. Roussel, Highlighting the risk of occupational exposure to hazardous drugs in the health care setting. Pharmacy Times, 2018.

3. Eisenberg, S., A Call to Action for Hazardous Drug Safety: Where We Have Been and Where 
We Are Now. Clinical journal of oncology nursing, 2016. 20(4).

4. Bernabeu-Martínez, M.A., et al., Guidelines for safe handling of hazardous drugs: A systematic review. PloS one, 2018. 13(5): p. e0197172.

5. Knobf, M., et al. The 2014-2018 Oncology Nursing Society Research Agenda. in Oncology nursing forum. 2015.

6. Eisenberg, S., Hazardous Drugs and USP $<$ $800>$ Implications for nurses. Clinical journal of oncology nursing, 2017. 21(2).

7. Power, L.A. and J.W. Coyne, ASHP guidelines on handling hazardous drugs. American Journal of Health-System Pharmacy, 2018. 75(24): p. 1996-2031.

8. Connor, T.H., et al., NIOSH list of antineoplastic and other hazardous drugs in healthcare settings 2016. 2016.

9. Celano, P., et al., Safe handling of hazardous drugs: ASCO standards. Journal of Clinical Oncology, 2019. 37(7): p. 598-609.

10. McGovern, P.M., et al., Factors affecting universal precautions compliance. Journal of Business and Psychology, 2000. 15(1): p. 149161.

11. Polovich, M. and P.C. Clark. Factors influencing oncology nurses' use of hazardous drug safehandling precautions. in Oncology Nursing Forum. 2012.

12. He, B.Y., et al. Personal protective equipment use and hazardous drug spills among ambulatory oncology nurses: Results from a mailed survey. in Oncology nursing forum. 2017. NIH Public Access.

13. Moher, D., et al., Preferred reporting items for systematic reviews and meta-analyses: the PRISMA statement. Int J Surg, 2010. 8(5): p. 336-341.
14. Polit, D.F. and C.T. Beck, Essentials of nursing research. Appraising evidence for, 2014.

15. Patel, K. and M. Pajpani, Making sense of the literature: an introduction to critical appraisal for the primary care practitioner. British Dental Journal, 2020. 229(8): p. 551-555.

16. Ciliska, D., S. Miccouci, and M. Dobbins, Effective public health practice project. quality assessment tool for quantitative studies. Hamilton, On: Effective Public Health Practice Project, 1998.

17. Thomas, B., et al., Quality assessment tool for quantitative studies dictionary: the Effective Public Health Practice Project (EPHPP). McMaster University, 2008.

18. Thomas, B., et al., A process for systematically reviewing the literature: providing the research evidence for public health nursing interventions. Worldviews on Evidence $\square$ Based Nursing, 2004. 1(3): p. 176-184.

19. Mun, M.Y. and S.Y. Hwang, Development and Evaluation of a Web-based Learning Course for Clinical Nurses: Anticancer Chemotherapy and Nursing. Korean Journal of Adult Nursing, 2020. 32(4): p. 364-373.

20. Keat, C.H., et al., Improving safety-related knowledge, attitude and practices of nurses handling cytotoxic anticancer drug: pharmacists' experience in a general hospital, Malaysia. Asian Pacific Journal of Cancer Prevention, 2013. 14(1): p. 69-73.

21. Shetaia, S., W. Shereif, and E. Omran, CHEMOTHERAPY SAFETY PROTOCOL FOR ONCOLOGY NURSES: IT'S EFFECT ON THEIR PROTECTIVE MEASURES PRACTICES. Mansoura Nursing Journal, 2017. 4(1): p. 267-279.

22. Friese, C.R., et al. Randomized controlled trial of an intervention to improve nurses' hazardous 
112 Medico-legal Update, January-March 2022, Vol.22, No. 1

drug handling. in Oncology nursing forum. 2019.

23. Crickman, R. and D.S. Finnell, Chemotherapy Safe Handling. Clinical journal of oncology nursing, 2017. 21(1).

24. Aebersold, M.L., et al., Evaluation of an Interprofessional Training Program to Improve Cancer Drug Therapy Safety. JCO Oncology Practice, 2021: p. OP. 20.00816.

25. Mohsen, M.M. and M.E. Fareed, Chemotherapy safety protocol for oncology nurses: It's effect on their protective measures practices. International Journal of Medical, Health, Biomedical,
Bioengineering and Pharmaceutical Engineering, 2013. 7(9): p. 529-37.

26. SAMIR, M.M., et al., EFFECT OF A DESIGNED TEACHING PROGRAM ON SAFE HANDLING OF CHEMO-THERAPY AMONG NURSES IN A SELECTED ONCOLOGY SETTING CAIROEGYPT. 2016.

27. Rai, D.K.S., et al., Impact of educational intervention on knowledge regarding safe handling of cytotoxic drugs among the nursing personnel working in BPKIHS. Health Renaissance, 2015. 13(1): p. 13-22. 